\title{
Risk factors in Angiographically Proven Coronary Patients
}

\author{
B. Rawat, Minalma Pandey, Ramana Acharya, Archana Satual, H.5, \\ Sodhi, Sunil C. Jha. Prof D.B. Karki, R.R. Kasliwal, Praveen Chandra, \\ Rajib Pande
}

\section{Background:}

CAD is coming as an epidemic in south east Asian countries. Yet recently coronary interventions have been almost non-existent in the Himalayan Kingdom of Nepal. To assess the local profile of the coronary disease, identify and establish the locally prevalent risk factors, coronary angiographic evaluation is mandatory. We recently completed 700 coronary angiograms in our institute.

\section{Methods:}

The equipment used was GE 1k workstation, CE0366 version 4.1 microlab, C-ARM series 9800 and angiomat illumena power injector. After the completion of 700 coronary angiograms a retrospective analysis was done regarding the age and gender distribution, result of angiography and complications and prevalence of Coronary risk factors.

\section{Results:}

Of 700 cases, 479 were males $(68 \%)$ and $221\{32 \%\}$ females.

651 pts $<70$ years, 49 pts $>70$ years

Normal angiogram $=259\{37 \%\}$

Single vessel disease $=53\{21.85 \%\}$

Double vessel disease $=116\{16.57 \%\}$

Triple vessel disease $=92\{13.14 \%\}$

Non critical $=77\{11 \%\}$

Left main artery disease $=13\{1.85 \%\}$ 
The risk factors amongst the patients with angiography proven coronary disease were as follows:

1. HTN : $322\{73.01 \%\}$

2. Smoking: $290\{65.75 \%\}$

3. Dyslipidemia : $144\{32.65 \%\}$

4. Diabetes: $150\{34.01 \%\}$

5. Positive family history: $78\{17.68 \%\}$

\section{Conclusions:}

Smoking and Hypertension are the commonest risk factors amongst the coronary angiographically proven patients in Nepal. An aggressive preventive approach directed towards these two risk factors may help in decreasing the expensive burden of coronary disease in this poor country of south Asia.

Norvic: Escorts International Hospital, Kathmandu, Nepal. 